transcribed spacer region was identical to that of the Trichophyton rubrum (BCCM/IHEM strain IHEM_27197) sequence in GenBank (accession no. MK299089.1). We treated the patient with oral terbinafine (125 mg/tablet) and the nail lesions improved (Fig. 1e). Treatment was stopped after 3 months.

In this case, C. cladosporioides, a chromogenic and common fungus, was detected in the culture of the removed nail. T. rubrum was detected using PCR amplification. This finding suggests that the white onychomycosis was due to T. rubrum. We suspected that the white ring fingernail lesion became infected from his foot.

In the 2019 report in the Japanese Journal of Dermatology, the Japanese Dermatological Association presented guidelines for treating dermatophytosis. ${ }^{1}$ The guidelines concerning onychomycosis are beneficial and suitable for recent oral and topical treatments. The classification of clinical onychomycosis manifestations in these guidelines is the same as the international classification by the British Association of Dermatologists. $^{2}$

Endonyx onychomycosis (EO) is rarely observed ${ }^{3,4}$ and is not a popular diagnosis in Japan because the previous Japanese classification system for onychomycosis excluded EO. Tosti et al. ${ }^{4}$ originally identified EO as being caused by Trichophyton soudanense, and describe EO as follows: "Clinically characterized by a diffuse milky-white discolouration of the affected nail, in the absence of nail bed hyperkeratosis or onycholysis. Nail plate surface and nail thickness is normal." The case presented here fulfilled these clinical characteristics. To date, EO has been caused by $T$. soudanense, Trichophyton violaceum, Trichophyton tonsurans and T. rubrum. Mulvaney et al..$^{5}$ reported that EO was resistant to standard oral and topical therapies; however, in this case removing a major portion of the affected nail and treating the patient with oral terbinafine was very effective.

Japanese dermatologists should review the guidelines for dermatophytosis and be vigilant for EO during their daily medical practices.

CONFLICT OF INTEREST: None declared.

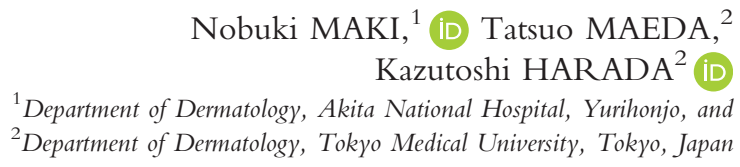

doi: 10.1111/1346-8138.15326

\section{REFERENCES}

1 Mochizuki T, Tsuboi R, lozumi $\mathrm{K}$ et al. Japanese dermatological association guidelines: guidelines for the management of dermatophytosis 2019. Jpn J Dermatol 2019; 129: 2639-2673.

2 Ameen M, Lear JT, Madan V, Mohd Mustapa MF, Richardson M. British Association of Dermatologists' guidelines for the management of onychomycosis 2014. Br J Dermatol 2014; 171: 937-958.

3 Harada T. Tinea unguium. Med Mycol J 2011; 52: 77-95.

4 Tosti A, Baran R, Piraccini BM, Fanti PA. Endonyx onychomycosis: a new modality of nail invasion by dermatophytes. Acta Derm Venereol (Stockh) 1999; 79: 52-53.

5 Mulvaney PM, Telang GH, Jellinek N. Trichophytum rubrum endonyx onychomycosis resistant to standard oral and topical therapies. Dermatol Online J 2015; 21 (9).

\title{
Clouston syndrome with pili canaliculi, pili torti, overgrown hyponychium, onycholysis, taurodontism and absence of palmoplantar keratoderma
}

\section{Dear Editor,}

An 11-year-old Thai boy presented with impaired hair growth characterized by sparse, fine and pale hair. The hair growth impairment was more pronounced within the vertex area. At birth, he had normal hair growth, which progressively arrested, thus leading to a short-hair phenotype (Fig. 1a). Intelligence, sweating and hearing were unremarkable. Fingernails were narrow and convex with overgrown hyponychium. The free edges of the fingernails detached from the nail beds and were located far inwards from the tips of the fingers. Toenails were convex (Fig. 1b,c), while palms and soles were normal. Teeth were clinically normal as well. A panoramic radiograph revealed taurodontism of the maxillary first permanent molars (Fig. 1k).
The scalp hair was extremely fine. Scanning electron microscopy of scalp hair showed pili canaliculi, pili torti and abnormal cuticles. Most hair showed twisted hair shafts near the hair follicles. No evidence of breaks of the hair shafts was found. Lack of hair cuticles was observed in many areas along the shafts (Fig. 1d-i). Amorphous material with numerous pits appeared to coat the hair shafts (Fig. 1j). In situ hybridization detected Gjb6 expression in the matrix progenitors of developing hair germs (Fig. 1l,m).

Following approval of the ethics committee, obtaining informed consent and in full accordance with the Declaration of Helsinki, whole-exome sequencing was performed on the patient's DNA. A de novo GJB6 mutation (c.31G $>A$;

Correspondence: Piranit Kantaputra, D.D.S., M.S., Division of Pediatric Dentistry, Department of Orthodontics and Pediatric Dentistry, Faculty of Dentistry, Chiang Mai University, 239 Huaykaew Road, Suthep, Mueang Chiang Mai District, Chiang Mai 50200, Thailand. Email: dentaland17@gmail.com 
Figure 1. (a) An 11-year old Thai boy with sparse, fine, pale and slow-growing hair. (b) Fingernails are narrow, convex with overgrown hyponychium. The free edges of the fingernails detach from the nail beds, which are located far inwards from the tips of the fingers. (c) Convex toenails. Scanning electron micrographs of hair from the top of the head. (d) Hair shaft showing $180^{\circ}$ twist covered with an amorphous coating. (e,f) Twisted and abnormal hair follicles. Scanning electron micrographs of scalp hair from the occipital area. (g) Very fine hair with marked pili torti with pili canaliculi. (h) Hair shaft shows marked twisted hair follicle with indistinct cuticular pattern. (i) The hair shaft near the root end is twisted $180^{\circ}$ and the root end is very abnormal with flattening and then grooving and splitting. There is some evidence of the cuticle showing through the coating. (j) Detail of the coating material covering the hair showing numerous pits and masking the presence or absence of cuticular cells. (k) Panoramic radiograph shows taurodontism (vertically long pulp chamber with shortened root length) (arrows). (I) Histology section staining with hematoxylineosin showing developing hair at E14 (original magnification $\times 200)$. $(\mathrm{m})$ Serial section of that in (I). In situ hybridization with $\left[{ }^{35} \mathrm{~S}\right]$ UTP-labeled riboprobes showing Gjb6 expression in developing hair matrix.

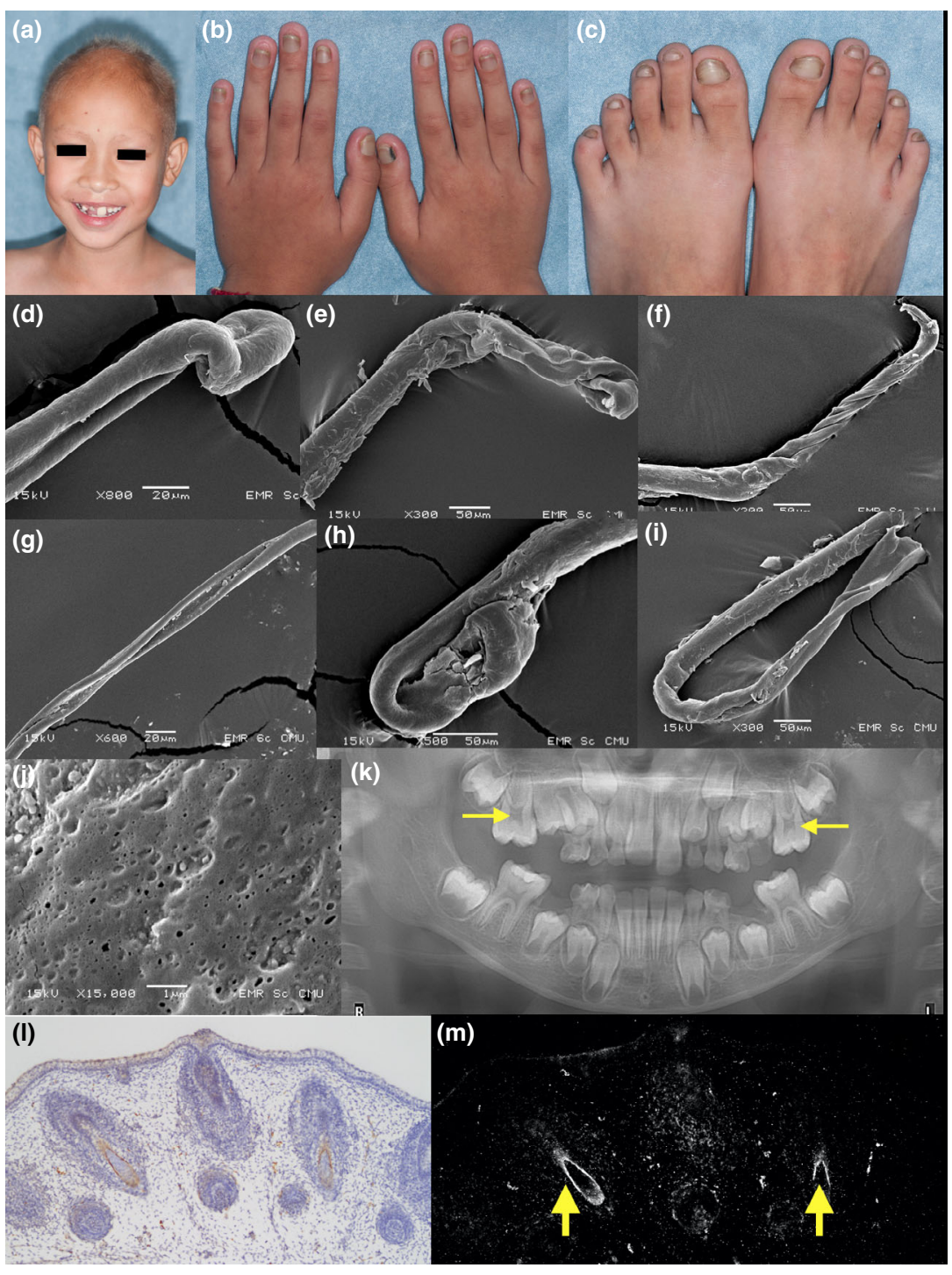

p.Gly11Arg) was identified in the patient, but not in his parents (Figure S1). This identified variant is known to be associated with Clouston syndrome (Mendelian Inheritance in Man \#129500), which is a rare autosomal dominant genodermatosis characterized by sparse, fine, pale, brittle and slow-growing hair, abnormal nails and palmoplantar keratoderma. ${ }^{1,2}$

The whole-exome sequencing results excluded the effects of other known dental anomaly-associated genes including WNT10A, WNT10B, MSX1, AXIN2, PAX9, GREM2 and $T F A P 2 B .{ }^{3}$ Therefore, taurodontism in the patient was likely the result of GJB6 mutation.

The detachment of the fingernail plates from the nail beds found in our patient resembled trauma-induced onycholysis, thus suggesting that GJB6 may play a role in the natural attachment between the nail plate and the nail bed. Overall, it appears that the developmental onycholysis observed in the patient is an outcome of GJB6 mutation. Ultrastructurally, almost all hair appeared twisted. It is hypothesized that gap junctions are important for straightness of hair, because a mutation in a gap junction gene, GJB6, apparently led to twisted hair follicles and shafts.

Dental anomalies have been reported only in Thai patients who carried p.Ala88Val ${ }^{1}$ and p.Gly11Arg mutations, ${ }^{2}$ which would suggest a link to their racial/genetic background. ${ }^{2}$ Noteworthy, GJB6 is a direct target gene of TP63 and TP63, known to play an important role in tooth, nail and hair development. ${ }^{4,5}$ This would explain why patients carrying GJB6 mutations have dental, nail and hair abnormalities. This and our previous report show that patients with Clouston syndrome do not need to have palmoplantar keratoderma, ${ }^{2}$ and that dental anomalies, pili canaliculi, pili torti, abnormal hair follicles and developmental onycholysis are also characteristic features of Clouston syndrome.

ACKNOWLEDGMENTS: We thank our patients and their families for their kind cooperation and for allowing us to use their 
medical and dental information for the benefit of others. This work was supported by the Thailand Research Fund; the Dental Association of Thailand; and the Faculty of Dentistry, Chiang Mai University.

CONFLICT OF INTEREST: None declared.

Piranit KANTAPUTRA, ${ }^{1,2}$
Worrachet INTACHAI, ${ }^{1}$ Katsushige KAWASAKI,
Atsushi OHAZAMA, ${ }^{3}$ Bruce CARLSON,
Natalina QUARTO, ${ }^{5}$ Chulabhorn PRUKSACHATKUN,
Mati CHUAMANOCHAN ${ }^{7}$ iD
${ }^{1}$ Division of Pediatric Dentistry, Department of Orthodontics and Pediatric
Dentistry, Faculty of Dentistry, Chiang Mai University, ${ }^{2}$ Dentaland Clinic,
Chiang Mai, Thailand, ${ }^{3}$ Division of Oral Anatomy, Department of Oral
Biological Science, Niigata University Graduate School of Medical and Dental
Sciences, Niigata, Japan, ${ }^{4}$ Department of Anatomy and Cell Biology,
University of Michigan, Ann Arbor, Michigan, ${ }^{5}$ Division of Plastic and
Reconstructive Surgery, Department of Surgery, Stanford University School of
Medicine, Stanford, California, USA, ${ }^{6}$ Division of Pediatric Dermatology,
Department of Pediatrics, Faculty of Medicine, and ${ }^{7}$ Division of Dermatology,
Department of Internal Medicine, Chiang Mai University, Chiang Mai,
Thailand

doi: $10.1111 / 1346-8138.15333$

\section{REFERENCES}

1 Sukakul T, Yang HS, Onoufriadis A, Hsu CK, McGrath JA. Pterygium and thinning of nails as an unusual manifestation in Clouston syndrome. J Dermatol 2019; 46: e329-e330.

2 Kantaputra PN, Intachai W, Carlson BM, Pruksachatkunakorn C. Clouston syndrome with dental anomalies, micropores of hair shafts, and absence of palmoplantar keratoderma. $J$ Dermatol 2020; 47: e90-e91.

3 Kantaputra PN, Hutsadaloi A, Kaewgahya $\mathrm{M}$ et al. WNT10B mutations associated with isolated dental anomalies. Clin Genet 2018; 93: 992-999.

4 Fujimoto A, Kurban M, Nakamura M et al. GJB6, of which mutations underlie Clouston syndrome, is a potential direct target gene of p63. J Dermatol Sci 2013; 69: 159-166.

5 Kantaputra PN, Hamada T, Kumchai T, McGrath JA. Heterozygous mutation in the SAM domain of p63 underlies Rapp-Hodgkin ectodermal dysplasia. J Dent Res 2003; 82: 433-437.

\section{SUPPORTING INFORMATION}

Additional Supporting Information may be found in the online version of this article:

Figure S1. Electropherograms of the de novo GJB6 variant (c.31G $>A$; p.Gly11Arg) in the patient but not in the parents.

\section{Partial spontaneous regression of pediatric large fibroblastic connective tissue nevus}

Dear Editor,

Fibroblastic connective tissue nevus (FCTN) is a newly recognized, benign, cutaneous mesenchymal lesion of fibroblastic/myofibroblastic lineage. Herein, we present the case of a patient with large FCTN associated with partial spontaneous remission over a 3-year follow-up period.

A 9-month-old boy presented with an erythematous plaque on the right side of his back, which had been present since birth. Physical examination revealed an asymmetrical whitish plaque measuring $4 \mathrm{~cm} \times 3 \mathrm{~cm}$, with telangiectasia in the center. Several round, soft, well-demarcated nodules of normal color were observed surrounding the main plaque (Fig. 1a,b). Dermatoscopic examination revealed a whitish pink structureless pattern and reticular telangiectasia (Fig. 1c). Histopathological examination revealed a poorly circumscribed proliferation of spindle cells arranged in short-to-longer intersecting fascicles entrapping appendage structures (Fig. 1d). Remarkable acanthosis and elongated vessels were observed in the dermal papillary layer. The spindle cells exhibited fibroblastic features with pale eosinophilic cytoplasmic extensions and inconspicuous nucleoli (Fig. 1e). Immunohistochemical study demonstrated weak positivity for CD34 (Fig. 1f) and focal positivity for smooth muscle actin on spindle cells (Fig. 1g), which were negative for desmin and S100. The overall features were consistent with FCTN diagnosis. The boy's parents did not consent to surgery. Over a 3-year period, the lesion flattened and became pigmented (Fig. 1h). Instead of whitish pink structureless pattern and telangiectasia, reticular pigmentation was noted on dermatoscopic examination (Fig. 1i).

Fibroblastic connective tissue nevus is a rare cutaneous lesion of fibroblastic/myofibroblastic disease and predominately arises on the trunk, head, neck and limbs of young girls. ${ }^{1}$ Most patients present with a solitary, slowly growing, painless plaque-like or nodular skin lesion. ${ }^{1}$ FCTN has been previously reported as a small nodule of less than $2.5 \mathrm{~cm} .{ }^{1-3}$ Our case showed a large whitish plaque measuring $4 \mathrm{~cm} \times 3 \mathrm{~cm}$, which was present since birth. The lesion is characterized by a deep, dermal proliferation of fibroblastic/myofibroblastic spindle cells with pale eosinophilic cytoplasm arranged in short intersecting fascicles entrapping appendages and dermal collagen bundles. The primary differential diagnosis of FCTN includes dermatomyofibroma (DMF), which is predominantly localized in the upper trunk and neck of young women. Histologically, fascicles of spindle cells in DMF are typically seen in parallel to the epidermis. ${ }^{4}$ On the other hand, spindle cell fascicles in FCTN exhibit a disorderly orientation relative to the epidermis. Immunologically,

Correspondence: Mika Takahashi, M.D., Department of Dermatology, Hirosaki University Graduate School of Medicine, 5 Zaifu-cho, Hirosaki, Aomori 036-8562, Japan. Email: mtakahashi@hirosaki-u.ac.jp 